\title{
Models of pollutant transport in natural aquifers
}
Amjad $\mathrm{Ali}^{1}$
Winston L Sweatman ${ }^{2}$
Robert McKibbin ${ }^{3}$

Received 15 November 2017; Revised 10 January 2018

\begin{abstract}
We present an approach for modelling the transport of pollution within a groundwater aquifer. The model is based on vertical discretisation of the aquifer into a number of horizontal layers. The approach can be used in a variety of situations. In particular, aquifers with lenses and phreatic surfaces are discussed. The use of the model is illustrated with examples including a comparison with experimental field data and the use of a second transported substance for remediation of the pollution.
\end{abstract}

Subject class: $86-\mathrm{XX}$

Keywords: Groundwater transport;modelling pollution

DoI:10.21914/anziamj.v59i0.12632, (C) Austral. Mathematical Soc. 2018. Published April 30, 2018, as part of the Proceedings of the 13th Biennial Engineering Mathematics and Applications Conference. ISSN 1445-8810. (Print two pages per sheet of paper.) Copies of this article must not be made otherwise available on the internet; instead link directly to the DOI for this article. 


\section{Contents}

1 Introduction

C50

2 Groundwater flow in an aquifer

C50

3 Pollutant transport and alleviation

C54

4 Illustrations

C55

5 Concluding comments

C60

References

C61

\section{Introduction}

There is a long history of study of groundwater transport in porous media [8, $10,7,9]$. The subject remains topical, with continuing concern about the management of these resources. Our models for pollutant transport are founded on the assumption that the natural layers present in aquifers are relatively homogeneous $[15,16,2,3,4,5,6,1]$. Here, we assemble formulae with which to model a variety of circumstances. We present examples that illustrate the effectiveness of the model in several different situations.

\section{Groundwater flow in an aquifer}

Our models divide the vertical profile of an aquifer into $\mathrm{N}$ thin homogeneous horizontal layers. This is partially motivated by the physically distinct strata created by geological formation. However, natural strata may be modelled using multiple model layers for more vertical detail. 
The physical dimensions of aquifers are disparate. Vertical thickness is very small in comparison with the horizontal extent or width [9]. We assume physical properties, such as porosity $\phi$ and permeability $K$, do not vary significantly with height within a layer. Any vertical differences are represented by interlayer variation. Layer properties only vary slowly with horizontal position $(x, y)$. The thickness and height of layers may change provided this is gradual so that gradients are slight.

We assume the groundwater is isothermal and incompressible and that the porous structure does not deform with time. Groundwater flow is found using Darcy's Law [11] together with the conservation of mass. The Darcy velocity $\mathbf{u}$ and the dynamic pressure $P$ satisfy

$$
\mathbf{u}=(\mathfrak{u}, v, w)=-\frac{K}{\mu} \nabla P, \quad \text { where } \quad P=p-p_{d}+\rho g\left(z-z_{d}\right),
$$

$\mu$ is dynamic viscosity, $\rho$ is fluid density, $g$ is gravitational acceleration, $p$ is absolute pressure, $z$ is height, and $p_{\mathrm{d}}$ and $z_{\mathrm{d}}$ are constant datum values.

A subscript $i$ is used to indicate values for the ith layer. An overbar denotes vertically averaged properties across the layer thickness $h_{i}=z_{i}-z_{i-1}$. The ith layer's volume flux vector

$$
\mathbf{q}_{\mathrm{i}}=\left(\mathbf{q}_{\mathrm{x}_{\mathrm{i}}}, \mathbf{q}_{y_{\mathrm{i}}}, \mathbf{q}_{z_{\mathrm{i}}}\right)=\int_{z_{\mathrm{i}-1}}^{z_{\mathrm{i}}} \mathbf{u d} z=-\frac{\mathrm{K}_{\mathrm{i}}}{\mu} \int_{z_{\mathrm{i}-1}}^{z_{\mathrm{i}}} \nabla \mathrm{Pd} z
$$

The Darcy velocity normal to the interface from the ith layer to the the $i+1$ th layer is $r_{i}=\mathbf{u}_{i} \cdot \nabla\left\{z-z_{i}(x, y)\right\}$. Conservation of mass requires $\nabla \cdot \mathbf{u}=0$ unless there is a source or sink term, such as due to a bore. If we include a volume flux source term $\bar{f}_{i}$, the conservation of mass leads to

$$
\nabla \cdot \mathbf{q}_{i}=r_{i-1}-r_{i}+h_{i} \bar{f}_{i} .
$$

For many modelling situations $\bar{f}_{i}=0$ at most, or perhaps all, locations.

Differentiating $h_{i} \bar{P}_{i}=\int_{z_{i-1}}^{z_{i}} P(x, y, z) d z$, and using equation (1),

$$
h_{i} \frac{\partial \bar{P}_{i}}{\partial x}=-\frac{\mu}{K_{i}} h_{i} \bar{u}_{i}+\left(P_{i}-\bar{P}_{i}\right) \frac{\partial z_{i}}{\partial x}-\left(P_{i-1}-\bar{P}_{i}\right) \frac{\partial z_{i-1}}{\partial x},
$$


where $P_{i}$ is the pressure at $z_{i}$. Thus, within the ith layer, the horizontal $x$-component of volume flux per unit width

$$
\mathrm{q}_{x_{i}}=h_{i} \bar{u}_{i}=\frac{K_{i}}{\mu}\left[-h_{i} \frac{\partial \bar{P}_{i}}{\partial x}+\left(P_{i}-\bar{P}_{i}\right) \frac{\partial z_{i}}{\partial x}-\left(P_{i-1}-\bar{P}_{i}\right) \frac{\partial z_{i-1}}{\partial x}\right] .
$$

The horizontal $y$-component is similar. The vertical component

$$
\mathrm{q}_{z_{i}}=h_{i} \bar{w}_{i}=\frac{K_{i}}{\mu}\left(P_{i-1}-P_{i}\right)
$$

Suppose that the horizontal Darcy velocity $(u, v)$ has magnitude $U$. Set $\epsilon=h / L$ as the ratio of thickness $h=z_{N}-z_{0}$ and horizontal lengthscale L. For positions without source terms, $\partial w / \partial z=-\partial u / \partial x-\partial v / \partial y$, by conservation of mass. Considering the magnitude of terms, $w$ is order $\epsilon \mathrm{U}$ and, from equation (6), P's vertical variation is order $(\mu / K) h \in U$. Hence the last two terms in equation (5) are of order $\epsilon^{2}$ smaller than the earlier terms and are ignored [3]. Also, dynamic pressure does not vary significantly with height and we replace subscripted values with P. The total volume flux per unit width

$$
\mathbf{q}(x, y)=\sum_{i=1}^{N} \mathbf{q}_{i}=-\frac{1}{\mu} \sum_{i=1}^{N} K_{i} h_{i} \nabla P .
$$

If an interface $\left(z=z_{i}\right)$ is impervious we have $r_{i}=0$. In particular a confined aquifer has impervious top and bottom surfaces $r_{0}=r_{N}=0$. Alternatively, there may be a phreatic top surface or a body of water such as a stream or lake may occupy the top surface [5]. By conservation of mass, if there are no sources or sinks or transmission across top or bottom surfaces of the aquifer, then $\nabla \cdot \mathbf{q}=0$.

Lenses A lens is a localised region where the physical medium properties differ from those in the surrounding aquifer. As before, interface slopes are 
gradual, so the lens thickness grows slowly inwards from zero at its edges. To model the lens, or a similar gradual vanishing of a physical layer within our layer-model, we allocate a set of layers to the lens. Their thicknesses are chosen as appropriate within the horizontal extent of the lens but everywhere else we take a negligible value $\delta>0$. This provides an accurate model in the limit $\delta \rightarrow 0$ while maintaining the number of layers [4].

Phreatic aquifers In a phreatic aquifer there is a free top surface $z=z_{\mathrm{t}}$, the phreatic surface, that gradually decreases in height in a downstream direction. At the phreatic surface the absolute pressure is approximately constant atmospheric pressure $p_{\text {atm }}$ and so, from equation (1),

$$
\mathrm{P}=\mathrm{p}_{\mathrm{atm}}-\mathrm{p}_{\mathrm{d}}+\rho \mathrm{g}\left(z_{\mathrm{t}}-z_{\mathrm{d}}\right) \text { and } \nabla \mathrm{P}=\rho \mathrm{g} \nabla z_{\mathrm{t}},
$$

and the specific discharge along the surface

$$
u_{s}=-\frac{K_{n} \rho g}{\mu} \frac{d z_{t}}{d s},
$$

where $\mathrm{n} \leqslant \mathrm{N}$ is the number of the layer currently containing the phreatic surface. The value of $n$ varies with horizontal position $(x, y)$. The slope of a phreatic surface is usually of order 0.001 to 0.01 [10] and we make the Dupuit assumption [12] replacing surface distance $s$ with horizontal distance within the derivative. From equations (7) and (8) we obtain the total volume flux

$$
\mathbf{q}(x, y)=-\frac{\rho g}{\mu}\left(\sum_{i=1}^{n-1} K_{i} h_{i}+K_{n}\left(z_{t}-z_{n-1}\right)\right) \nabla z_{t} .
$$

The phreatic surface is obtained from the conservation of mass. If there are no fluid sources or sinks

$$
\nabla \cdot\left[\left(\sum_{i=1}^{n-1} K_{i} h_{i}+K_{n}\left(z_{t}-z_{n-1}\right)\right) \nabla z_{t}\right]=0 .
$$




\section{Pollutant transport and alleviation}

Now consider a pollutant of concentration $c(x, y, z, t)$ (mass per unit volume of fluid) that is transported within the groundwater by advection and dispersion (through mechanical mixing and molecular diffusion). It is assumed that the pollutant does not affect the flow itself. The model [15] uses a Fickian-type dispersion with pollutant flux $\mathbf{q}_{\mathbf{c}}=\mathbf{c q}-\phi \mathrm{D} \nabla \mathbf{c}$, with $\mathrm{D}$ the dispersion tensor and $\phi$ the porosity of the aquifer. By conservation of pollutant mass

$$
\phi \frac{\mathrm{dc}}{\mathrm{dt}}=-\nabla \cdot \mathbf{q}_{\mathrm{c}}+\phi \mathrm{C}-\phi \gamma \mathrm{c}
$$

where the final two terms, involving $\mathrm{C}$ and $\gamma$, respectively, allow for the addition or removal of pollutant. We align the $x$-direction with the direction of the groundwater flow. In each layer we assume that the diagonal entries of the dispersion tensor $\mathrm{D}_{x_{\mathfrak{i}}}, \mathrm{D}_{y_{\mathfrak{i}}}$ and $\mathrm{D}_{z \mathfrak{i}}$ can be treated as constant, and that the vertical variation of concentration is modest and thus approximated by the mean concentration $\overline{\mathbf{c}}_{\mathfrak{i}}(x, y, t)$. Equation (12) becomes $[16,6]$

$$
\begin{aligned}
\phi_{i} h_{i} \frac{\partial \bar{c}_{i}}{\partial t}= & \frac{\partial}{\partial x}\left[-q_{x_{i}} \bar{c}_{i}+\phi_{i} h_{i} D_{x_{i}} \frac{\partial \bar{c}_{i}}{\partial x}\right]+\frac{\partial}{\partial y}\left[-q_{y_{i}} \bar{c}_{i}+\phi_{i} h_{i} D_{y_{i}} \frac{\partial \bar{c}_{i}}{\partial y}\right] \\
& +r_{i-1}^{+} \bar{c}_{i-1}+r_{i-1}^{-} \bar{c}_{i}+r_{i}^{+} \bar{c}_{i}+r_{i}^{-} \bar{c}_{i+1} \\
& +\tau_{i-1}\left(\bar{c}_{i-1}-\bar{c}_{i}\right)+\tau_{i}\left(\bar{c}_{i+1}-\bar{c}_{i}\right)+\phi_{i} h_{i} \bar{f}_{C i}-\phi_{i} h_{i} \bar{\gamma}_{i} \bar{c}_{i},
\end{aligned}
$$

where $\tau_{i}$ is the interlayer dispersive transfer coefficient given by

$$
\frac{1}{\tau_{i}}=\frac{h_{i}}{2 \phi_{i} D_{z i}}+\frac{h_{i+1}}{2 \phi_{i+1} D_{z i+1}},
$$

$r_{i}^{+}=\left(r_{i}+\left|r_{i}\right|\right) / 2$ and $r_{i}^{-}=\left(r_{i}-\left|r_{i}\right|\right) / 2$. The function $\bar{f}_{C i}$ is a layer-thicknessaveraged pollution source term at position $(x, y)$ in layer $i$. The form of the averaged removal coefficient $\bar{\gamma}_{i}$ depends on how the pollutant is removed. If we assume that pollutant is adsorbed onto the porous matrix itself and that this does not become saturated then we could treat $\bar{\gamma}_{i}$ as a constant. 

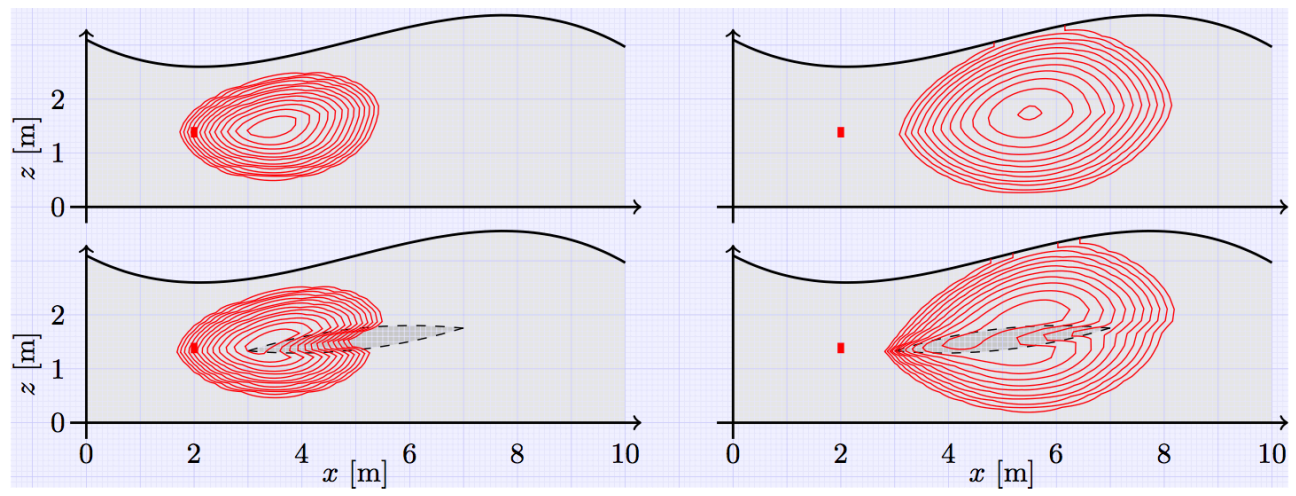

Figure 1: Pollutant concentration contours: (Top) Homogeneous aquifer, (Bottom) Aquifer with lens. (Left) Time 4 hours, (Right) Time 10 hours [1].

A special case of pollution removal is when the pollutant is removed by another substance that is also transported by the groundwater flow $[6,5]$. This remediation agent, which might be a strong oxidiser, can be injected downstream from a pollution event. The remediation agent has averaged layer concentration denoted $\bar{a}_{i}$, source term $\bar{f}_{A_{i}}$ and degradation rate $k_{A}$. In equation (13), the removal term has the form $-\phi_{i} h_{i} k_{C} \bar{a}_{i} \bar{c}_{i}$, i.e. $\bar{\gamma}_{i}=k_{C} \bar{a}_{i}$, where $k_{C}$ is the rate of degradation of pollutant due to the interaction with the remediation agent [6]. The remediation agent itself is governed similarly by equation (13), but with $\bar{a}_{i}, \bar{f}_{A i}$ and $k_{A}$ replacing $\bar{c}_{i}, \bar{f}_{C i}$ and $k_{C}$.

\section{Illustrations}

The effect of lenses We consider two aquifers, both having total volume flux per unit width $0.1 \mathrm{~m}^{2} \mathrm{~h}^{-1}$ travelling in the $x$-direction, porosity $\phi=0.1$ and dispersion coefficients $\mathrm{D}_{x}=0.03$ and $\mathrm{D}_{z}=0.005 \mathrm{~m}^{2} \mathrm{~h}^{-1}$. There is no $y$-direction variation in the aquifers or the pollutant release, and we use a two-dimensional model based upon equations (7), (13) and (14) with multiple 
sub-layers.

Figure 1 shows the aquifers four and ten hours after an instantaneous release of $1 \mathrm{~kg} \mathrm{~m}^{-1}$ pollutant per unit width in the layer containing $(x=2, z=1.4)$ at the position of the red rectangle. The pollutant concentration contours in all four plots are plotted on an equally-spaced logarithmic scale $10^{-3.00}$, $10^{-2.75}, 10^{-2.50}, \ldots, 10^{-0.00} \mathrm{~kg} \mathrm{~m}^{-3}$. The first aquifer is homogeneous with $\mathrm{K}=10^{-7} \mathrm{~m}^{2}$ and the second contains a lens with $\mathrm{K}=10^{-8} \mathrm{~m}^{2}$ and elsewhere has $\mathrm{K}=10^{-7} \mathrm{~m}^{2}$. The mean dynamic pressure at the lateral boundaries of the two aquifers is different to maintain the same volume flux. In the lower plot on the left, four hours after release, the point of maximum pollutant concentration has only just reached the left end of the lens. As the lens has lower permeability, the fluid and advected pollutant travels more slowly within it. This makes the lens initially appear more resistant to the pollutant and the rising contour lines lag behind those for similar $x$-distances in the main aquifer. Here, there is a net dispersion of pollutant into the lens from above and below. In the lower plot on the right, after ten hours, the same lagging effect can be seen in the rising contour lines on the right end of the lens. However, on the left end of the lens the falling contours also lag behind similar $x$-distances in the main aquifer. The slow fluid speeds within the lens are trapping pollutant within it. While slowly advecting horizontally through the lens, the pollutant will leak up and down into the main aquifer though dispersion. This will continue for some time after most of the pollutant has advected through the neighbouring higher permeability region.

A comparison with experimental field data The model is fitted to experimental field data collected at the Canadian Forces Base in Borden, Ontario [14]. For this a $12 \mathrm{~m}^{3}$ solution containing several tracers was injected over a 14.75-hour period at nine closely spaced well sites within a phreatic sand aquifer. The tracers included a mass $10.7 \mathrm{~kg}$ chloride ions and these are modelled here. The aquifer is relatively homogeneous with mean hydraulic conductivity $\rho \mathrm{gK} / \mu=7 \times 10^{-5} \mathrm{~m} \mathrm{~s}^{-1}$, hydraulic head gradient in the direction of flow $\nabla z_{\mathrm{t}}=-0.0043$, and measured total porosity $\phi_{\mathrm{tot}}=0.33$. The 


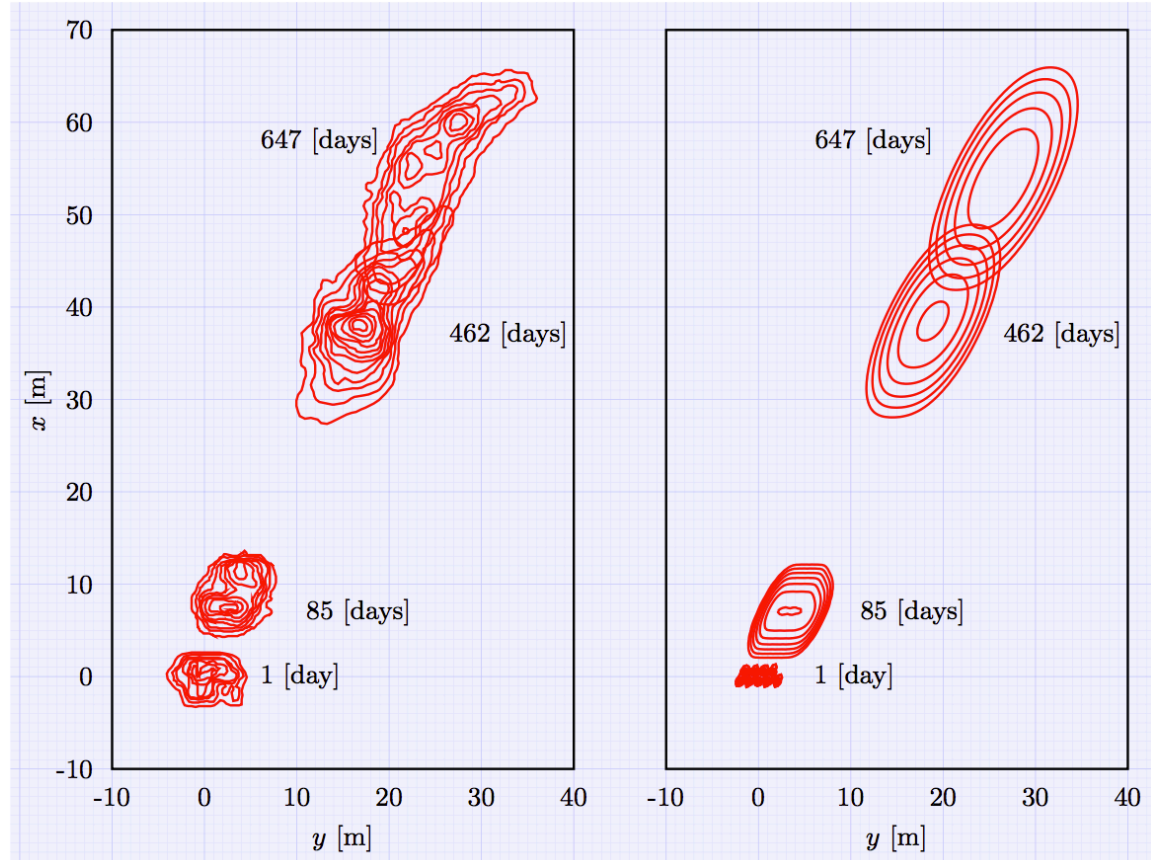

Figure 2: The vertically-averaged chloride concentration profile at 1, 85, 462, and 647 days after injection: (Left) Experiment [14], (Right) Model [1].

longitudinal dispersivity $\alpha_{\mathrm{L}}=\mathrm{D}_{\chi} /|\mathbf{q}|$ has been estimated to be $0.49 \mathrm{~m}$ [13]. The groundwater flow is assumed uniform and unidirectional. Using the measured values, taking $\phi=\phi_{\text {tot }}$, and applying equation (10), with a single layer, the model tracer velocity $\mathbf{q} /\left(z_{\mathrm{t}}-z_{0}\right) \phi$ has magnitude $0.079 \mathrm{~m}$ day $^{-1}$. This is slightly less than the measured value of $0.091 \mathrm{~m}_{\text {day }}{ }^{-1}$. However, the difference can be attributed to the effective porosity being lower than the total porosity and errors in the estimated data from the measurements [14].

Figure 2 compares experimental data with the model, using equations (10) and (13). The values of effective porosity $\phi$ and transverse dispersivity $\alpha_{\mathrm{T}}=\mathrm{D}_{\mathrm{y}} /|\mathbf{q}|$ have been fitted as parameters. The observed chloride ion 
concentration after 1, 85, 462 and 647 days are shown by contours on the left-hand side of Figure 2 [14]. These have been normalised by dividing by the maximum concentration. Model results, for the same times and similarly normalised, are shown on the right-hand side of the figure. The effective porosity $\phi$ is set at 0.28 . This is reasonably comparable with the total porosity value $\phi_{\text {tot }}=0.33$ but is in agreement with the measured tracer velocity. The transverse dispersivity $\alpha_{\mathrm{T}}$ is set at $0.05 \mathrm{~m}$. This can be compared with the longitudinal dispersivity $\alpha_{\mathrm{L}}=0.49 \mathrm{~m}$. The ratio $\alpha_{\mathrm{T}} / \alpha_{\mathrm{L}}$ can take a wide range of values [10]. The value for the model here is comfortably within this range

Pollution remediation Consider a confined aquifer of total thickness $10 \mathrm{~m}$ with three level strata of equal thicknesses, consisting of sand and gravel $\mathrm{K}=10^{-9} \mathrm{~m}^{2}$ sandwiched between two strata of clean sand $\mathrm{K}=10^{-10} \mathrm{~m}^{2}$. The strata are each divided into five model sub-layers also of equal thickness. The total groundwater flux per unit width is $10 \mathrm{~m}^{2}$ day ${ }^{-1}$ in the $x$-direction. There is no variation with $y$ as sources are assumed to be extended in this direction. Throughout the aquifer, the dispersion coefficients $D_{x_{i}}=0.2$ and $\mathrm{D}_{z \mathfrak{i}}=0.06 \mathrm{~m}^{2}$ day $^{-1}$ with porosity $\phi=0.1$. An instantaneous release of $1 \mathrm{~kg} \mathrm{~m}^{-1}$ pollutant per unit width occurs in the third model sub-layer from the top of the aquifer at $x=10 \mathrm{~m}$. The pollutant is advected, meanwhile dispersing horizontally and vertically. Pollutant entering the more permeable middle stratum is advected at a higher speed [6]. Some of this pollutant later disperses back into the upper stratum. We explore the effect of an instantaneous release of $1 \mathrm{~kg} \mathrm{~m}^{-1}$ remediating agent per unit width in a layer downstream of the original pollutant release and two days later. The degradation rates $k_{C}=2$ and $k_{A}=1\left(\mathrm{~kg} \mathrm{~m}^{-3}\right)^{-1}$ day $^{-1}$.

Equations (7), (13) and (14) apply. These indicate that $\mathbf{q}_{x_{i}}, \mathbf{u}_{x_{i}}, \mathbf{K}_{\boldsymbol{i}}$ and the speed of advection in a layer are all proportional. Hence the flow in the central stratum is ten times as high as that in the other strata. Further, the speed of advection in the top stratum is found to be $2.5 \mathrm{~m}_{\text {day }}{ }^{-1}$. In Figure 3, various scenarios are illustrated with two graphs per scenario. The upper plot for each of the scenarios shows pollutant mass still present in the aquifer (red), 


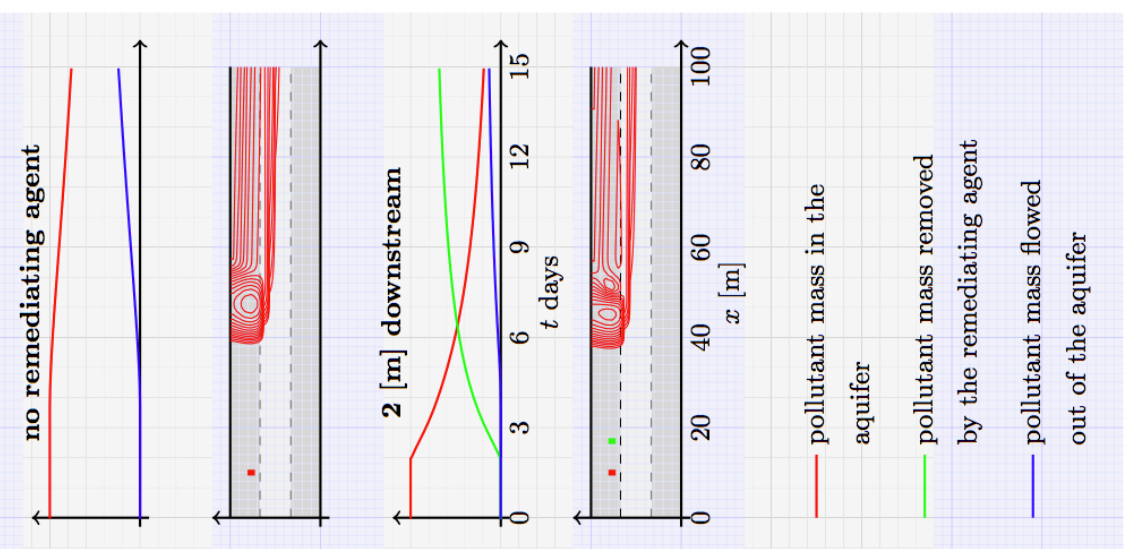

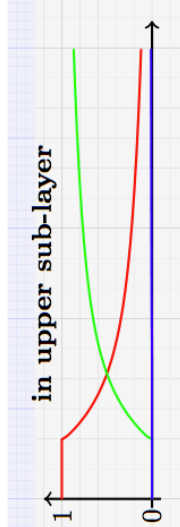

[8y] ssew

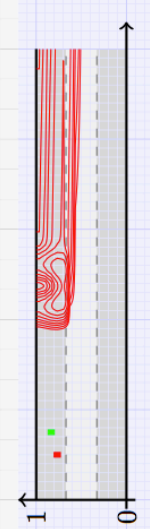

[w] $z$
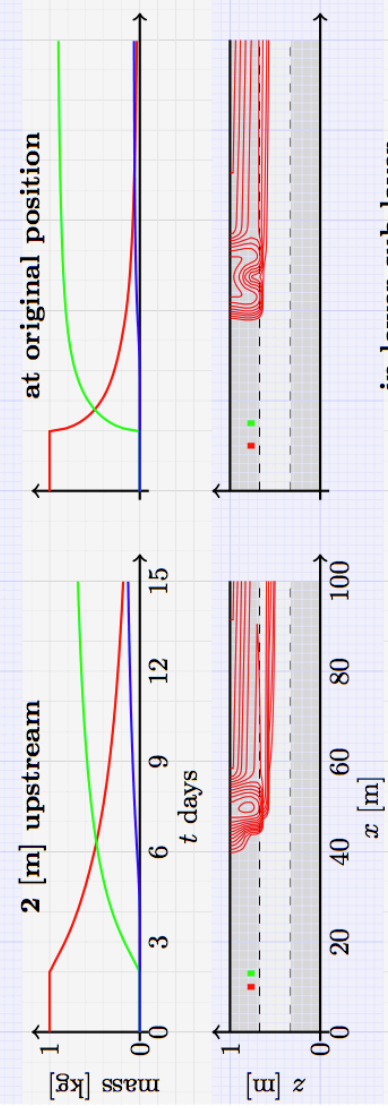

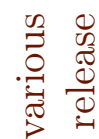

ते

ช 20

$\stackrel{0}{0} \stackrel{0}{0}$

范

ن

屯ु
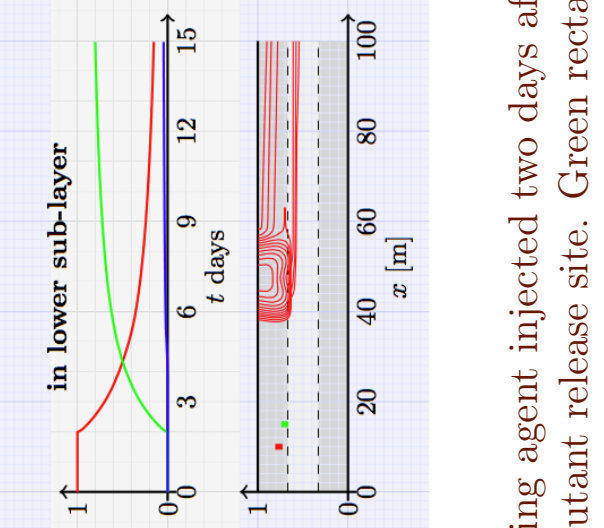

[8ิy] sseu $[\mathrm{w}] z$

8

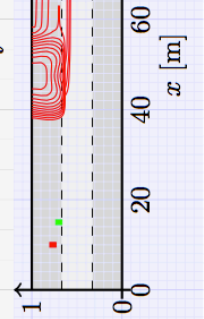

己ֶ.

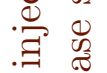

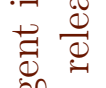

ఠి

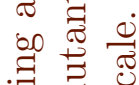

要

可 @

ฮี

+

प

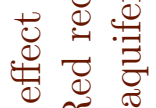

리 밈

نे 0

○.ำ

当 
pollutant removed by the remediating agent (green) and pollutant that has flowed past the downstream boundary of the aquifer $x>100 \mathrm{~m}$ (blue). These are plotted against time. At any time the sum of these masses is equal to the released mass of pollutant $\left(1 \mathrm{~kg} \mathrm{~m}^{-1}\right)$. The lower plot for each of the scenarios shows pollutant concentration profiles 15 days after pollutant release. The contours are plotted on an equally-spaced logarithmic scale $10^{-4.000}, 10^{-3.725}$, $10^{-3.450}, \ldots, 10^{-0.150} \mathrm{~kg} \mathrm{~m}^{-3}$. The site of pollutant release is shown with a red rectangle and that of remediation agent release with a green rectangle. In the basic scenario the remediation agent is released $5 \mathrm{~m}$ downstream of the site of pollutant release. In other scenarios the remediation agent is released one sub-layer $\left(\frac{2}{3} \mathrm{~m}\right)$ higher, one sub-layer lower, $2 \mathrm{~m}$ upstream and $2 \mathrm{~m}$ downstream from the previous position or it is not released at all.

The most effective pollutant removal is in the original scenario. In this scenario the remediation agent is released at the heart of the pollution plume at the point where the pollutant would advect had there been no dispersion.

\section{Concluding comments}

We have modelled pollutant transport in a groundwater aquifer. The models build upon the assumption that the aquifer is constructed from homogeneous layers. We collect together several equations involved. To begin the modelling process, initially, the fluid flow is found (Section 2). Then the transport of the pollutant, by advection and dispersion, is calculated (Section 3). In Section 4, the model equations have been used for three different illustrations. First, we show the effect of a lens, or region of different permeability, upon the spread of pollutant. Then, using realistic parameters, the model is fitted to experimental field data. It captures the qualitative features of the pollutant transport. Finally, we illustrate the effect of a remediating agent released to counteract a pollution event and the effect of different release points is explored. There is further discussion of such models and additional illustration in our previous work $[15,16,2,3,4,5,6,1]$. 


\section{References}

[1] A. Ali (2015) Simplified modelling of pollutant transport in naturally-layered aquifers, $\mathrm{PhD}$ Thesis, Massey University, NZ. https://mro.massey.ac.nz/handle/10179/6782 C50, C55, C57, C59, C60

[2] A. Ali, R. McKibbin, W.L. Sweatman (2012) Fluid flow and solute transport in unevenly-stratified groundwater aquifers, AIP Conference Proceedings, 1453, 11-16. doi:10.1063/1.4711146 C50, C60

[3] A. Ali, R. McKibbin, W.L. Sweatman (2012) Simplified modelling of pollutant transport in stratified groundwater aquifers, Proceedings of IEMSs 2012, 2739-2746. http://www.iemss.org/sites/iemss2012/ proceedings/I1_0675_Ali_et_al.pdf C50, C52, C60

[4] A. Ali, R. McKibbin, W.L. Sweatman (2012) Tracer transport: Appearing or disappearing sub-layer in a groundwater aquifer, In: G. Mallinson, ed., Proceedings of the 23rd International Symposium on Transport Phenomena (ISTP), The University of Auckland, NZ. C50, C53, C60

[5] A. Ali, R. McKibbin, W.L. Sweatman (2014) A simplified model for transport in aquifers, Proceedings of ICPM5, ECI Symposium Series. http://dc.engconfintl.org/porous_media_V/56 C50, C52, C55, C60

[6] A. Ali, W.L. Sweatman, R. McKibbin (2014) Pollutant transport and its alleviation in groundwater aquifers, Mathematics in Industry, 19, 53-58. doi:10.1007/978-3-319-05365-3_8 C50, C54, C55, C58, C60

[7] M.P. Anderson, J.A. Cherry (1979) Using models to simulate the movement of contaminants through groundwater flow systems, CRC Critical Reviews in Environmental Control, 9(2):97-156. doi:10.1080/10643387909381669, C50 
[8] J. Bear (1972) Dynamics of fluid in porous media Dover, New York. ISBN 100486656756 C50

[9] J. Bear, Y. Bachmat (1991) Introduction to modeling of transport phenomena in porous media, Kluwer. ISBN 978-94-009-1926-6 C50, C51

[10] J. Bear, A. Verruijt (1978) Modeling groundwater flow and pollution D. Reidel, Dordrecht, Holland. C50, C53, C58

[11] H.P.G. Darcy (1856) Les fontaines publiques de la ville de dijon, Technical report, Victor Dalmont, Paris. C51

[12] J. Dupuit (1863) Études théoriques et pratiques sur le mouvement des eaux dans les canaux découverts et à travers les terrains perméables, Dunod, Paris, 2nd edition. C53

[13] D.L. Freyberg (1986) A natural gradient experiment on solute transport in a sand aquifer: 2. Spatial moments and the advection and dispersion of nonreactive tracers, Water Resources Research, 22, 2031-2046. doi:10.1029/WR022i013p02031 C57

[14] D.M. Mackay, D.L. Freyberg, P.V. Roberts, J.A. Cherry (1986) A natural gradient experiment on solute transport in a sand aquifer: 1 . Approach and overview of plume movement, Water Resources Research, 22, 2017-2029. doi:10.1029/WR022i013p02017 C56, C57, C58

[15] R. McKibbin (2009) Groundwater pollutant transport: transforming layered models to dynamical systems, An. Şt. Univ. Ovidius Constanţa, Ser. Mat., 17(3), 183-196. http://www.emis.ams.org/journals/ ASUO/mathematics/pdf19/McKibbin.pdf C50, C54, C60

[16] R. McKibbin (2010) Some aspects of modelling pollution transport in groundwater aquifers, In: L.H. Weiranto, S. Pudjaprasetya, editors, Proceedings of CIAM 2010, 9-16, ITB, Indonesia. C50, C54, C60 


\section{Author addresses}

1. Amjad Ali, Institute of Natural and Mathematical Sciences, Massey University, Auckland, New ZeAland.

2. Winston L Sweatman, Institute of Natural and Mathematical Sciences, Massey University, Auckland, New Zealand. mailto:w.sweatman@massey.ac.nz

3. Robert McKibbin, Institute of Natural and Mathematical Sciences, Massey University, Auckland, New ZEAland.

mailto:r.mckibbin@massey.ac.nz 